\title{
What is a mental/psychiatric disorder? From DSM-IV to DSM-V
}

\author{
D. J. Stein ${ }^{1 *}$, K. A. Phillips ${ }^{2}$, D. Bolton ${ }^{3}$, K. W. M. Fulford ${ }^{4}$, J. Z. Sadler ${ }^{5}$ and K. S. Kendler ${ }^{6}$ \\ ${ }^{1}$ Department of Psychiatry, University of Cape Town, South Africa \\ ${ }^{2}$ Department of Psychiatry and Human Behavior, Brown University, USA \\ ${ }^{3}$ Psychology Department, Institute of Psychiatry, King's College London, UK \\ ${ }^{4}$ Departments of Philosophy and Psychiatry, Oxford University, UK \\ ${ }^{5}$ Department of Psychiatry, University of Texas Southwestern, USA \\ ${ }^{6}$ Department of Psychiatry, Medical College of Virginia, USA
}

The distinction between normality and psychopathology has long been subject to debate. DSM-III and DSM-IV provided a definition of mental disorder to help clinicians address this distinction. As part of the process of developing DSM-V, researchers have reviewed the concept of mental disorder and emphasized the need for additional work in this area. Here we review the DSM-IV definition of mental disorder and propose some changes. The approach taken here arguably takes a middle course through some of the relevant conceptual debates. We agree with the view that no definition perfectly specifies precise boundaries for the concept of mental/psychiatric disorder, but in line with a view that the nomenclature can improve over time, we aim here for a more scientifically valid and more clinically useful definition.

Received 15 September 2009; Revised 23 November 2009; Accepted 24 November 2009; First published online 20 January 2010

Key words: Mental disorder, nosology, philosophy, psychiatric disorder.

\section{Introduction}

DSM-III and DSM-IV have been praised for making a seminal contribution to patient care and to the scientific study of psychiatric disorders by providing rigorous and reliable diagnostic criteria for conditions such as major depressive disorder and social phobia. At the same time, DSM-III and DSM-IV have been criticized for creating too many diagnostic categories (van Praag, 2000) and for allowing the distinction between psychopathology and normal psychological phenomena (e.g. sadness after a major stressful event, shyness in social situations) to be eroded (Wakefield et al. 2005; Horwitz \& Wakefield, 2007). Both DSM-III and DSM-IV emphasized the difficulties inherent in drawing a precise distinction between normality and psychopathology, and they provided a definition of mental disorder that attempted to address this challenge (Spitzer \& Endicott, 1978). This issue is relevant to deciding not only whether a disorder should be in the nosology but also whether the criteria for a particular disorder are optimal for defining the threshold for caseness. As part of the process of developing DSM-V, researchers have explored again the concept

\footnotetext{
* Address for correspondence: Professor D. J. Stein, UCT Department of Psychiatry, Groote Schuur Hospital J-2, Anzio Rd, Observatory 7925, Cape Town, South Africa.

(Email: dan.stein@uct.ac.za)
}

of mental disorder and emphasized the need for additional work in this area (Rounsaville et al. 2002). A literature review on this topic has been commissioned by the DSM-V Anxiety, Obsessive-Compulsive Spectrum, Post-Traumatic, and Dissociative Disorders Work Group, and is in process. In this brief editorial, we review the DSM-IV definition of mental disorder and propose some changes. Recommendations provided in this editorial should be considered preliminary at this time; they do not necessarily reflect the final recommendations or decisions that will be made for DSM-V, as the DSM-V development process is ongoing.

\section{DSM-IV definition of mental disorder}

DSM-IV states that:

although this manual provides a classification of mental disorders, it must be admitted that no definition adequately specifies precise boundaries for the concept of 'mental disorder'. The concept of mental disorder, like many other concepts in medicine and science, lacks a consistent operational definition that covers all situations. All medical conditions are defined on various levels of abstraction - for example, structural pathology (e.g. ulcerative colitis), symptom presentation (e.g. migraine), deviance from a physiological norm (e.g. hypertension), and etiology (e.g. pneumococcal pneumonia). Mental disorders have also been defined by a variety of concepts (e.g. distress, dyscontrol, 
Table 1. DSM-IV definition of mental disorder

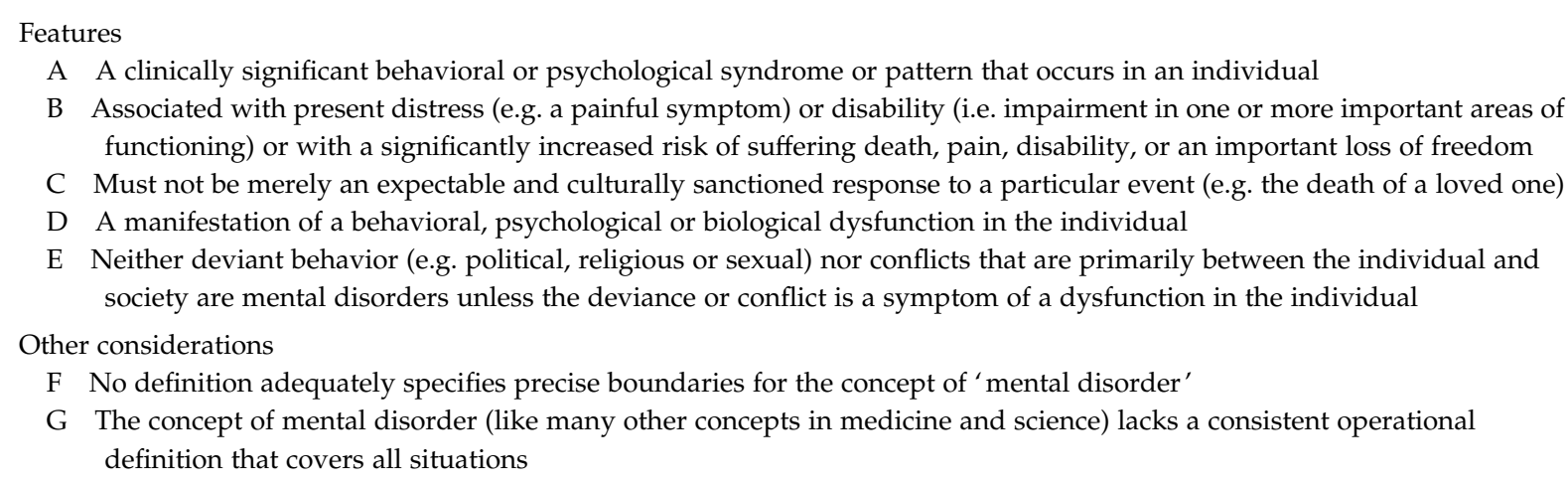

disadvantage, disability, inflexibility, irrationality, syndromal pattern, etiology, and statistical deviation). Each is a useful indicator for a mental disorder, but none is equivalent to the concept, and different situations call for different definitions.

DSM-IV goes on, however, to state that:

Despite these caveats, the definition of mental disorder that was included in DSM-III and DSM-III-R is presented here because it is as useful as any other available definition and has helped to guide decisions regarding which conditions on the boundary between normality and pathology should be included in DSM-IV. In DSM-IV, each of the mental disorders is conceptualized as a clinically significant behavioral or psychological syndrome or pattern that occurs in an individual and that is associated with present distress (e.g. a painful symptom) or disability (i.e. impairment in one or more important areas of functioning) or with a significantly increased risk of suffering death, pain, disability, or an important loss of freedom. In addition, this syndrome or pattern must not be merely an expectable and culturally sanctioned response to a particular event, for example, the death of a loved one. Whatever its original cause, it must currently be considered a manifestation of a behavioral, psychological, or biological dysfunction in the individual. Neither deviant behavior (e.g. political, religious, or sexual) nor conflicts that are primarily between the individual and society are mental disorders unless the deviance or conflict is a symptom of a dysfunction in the individual, as described above.

Table 1 operationalizes the DSM-IV definition of mental disorder in the standard format used for the operationalization of clinical diagnoses.

\section{Proposed DSM-V definition of mental/psychiatric disorder}

Table 2 presents our suggested changes. Before going on to provide a rationale for each of these changes, it is relevant to address the question of whether the term 'mental disorder' is optimal. 'Mental' implies a
Cartesian view of the mind-body problem, that mind and brain are separable and entirely distinct realms, an approach that is inconsistent with modern philosophical and neuroscientific views (Fulford et al. 2006). The term 'psychiatric disorder' may be preferable insofar as it emphasizes that these conditions are not purely 'mental' and that the line between 'psychiatric disorder' and 'other medical disorders' is not distinct. However, mental health clinicians other than psychiatrists have also criticized this term as it may suggest, incorrectly, that only psychiatrists are trained in the diagnosis and management of these conditions (Spitzer \& Williams, 1982). Such criticism may be sufficient to warrant retaining 'mental disorder', and indeed the authors of this article could not come to a consensus on this matter. One potential compromise is to recommend the awkward term 'mental/ psychiatric'. A more conservative approach would be to retain the term 'mental disorder', in keeping with DSM-IV, but to emphasize in the text that these are brain-mind disorders.

\section{Criterion $A$}

DSM-IV refers to a clinically significant behavioral or psychological syndrome or pattern that occurs in an individual. However, the phrase 'clinically significant' is in some ways tautological here; its definition is precisely what is at stake when defining a mental disorder. Other definitional criteria go on to tackle the meaning of clinical significance, and we therefore suggest omitting the 'clinically significant' phrase from criterion A. Nevertheless, the phrase 'clinically significant' is useful in defining a mental disorder, and we therefore turn to it in criterion B.

As alluded to earlier, the question has been raised of what is 'mental' about mental disorders (Fulford et al. 2006). In this regard, a practical issue that arises is the inclusion in DSM-IV of symptoms and disorders 
Table 2. DSM-V proposal for the definition of mental/psychiatric disorder

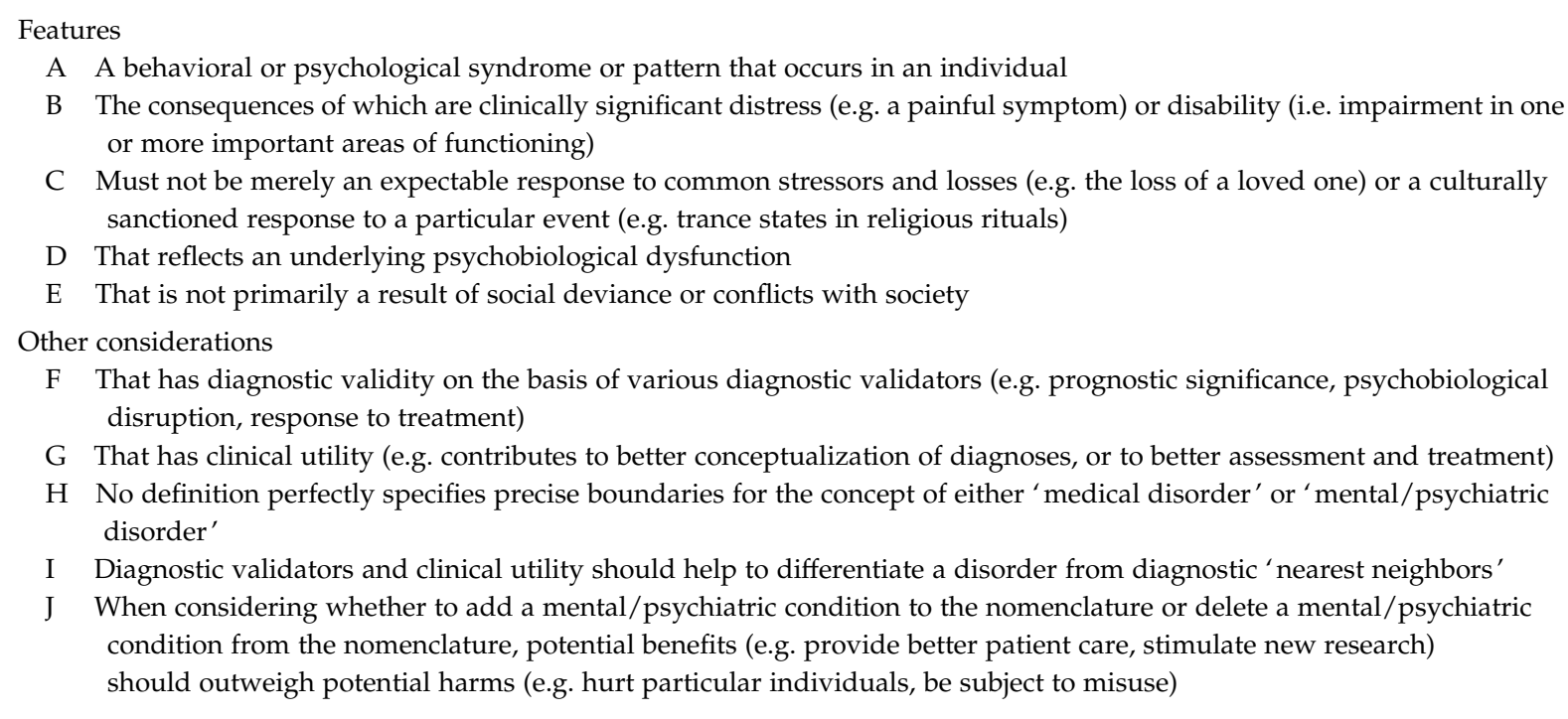

that might be conceptualized as more neurological (rather than behavioral or psychological) in nature (e.g. tic disorders, catatonia). It might be argued that involuntary motoric movements (or lack of motoric movement) belong in a classification of neurological disorders rather than mental/psychiatric disorders. However, the constructs 'voluntary' and 'involuntary' arguably have fuzzy borders. Furthermore, the term 'behavioral' in criterion A could be considered to cover motoric symptoms that lie in a border area between voluntary and involuntary, supporting the inclusion of conditions such as tic disorders in DSM-V.

Regarding the phrase 'in an individual' in criterion A, there has been debate about whether dysfunction in relationships should be classified as mental/ psychiatric disorders (Heyman et al. 2009). Although currently listed only as $\mathrm{V}$ codes (other conditions that may be a focus of clinical attention), such phenomena seem to have content validity, may be associated with significant distress and impairment, and can be reliably diagnosed. Nevertheless, general medical disorders invariably occur within individuals, and although there may be some reasons to stretch the construct of disorder to novel phenomena such as relationship dysfunction, such an expansion would necessarily be contentious and therefore would require particularly persuasive supporting data. We therefore suggest retaining the phrase 'occurs in an individual' at this time. (Notably, DSM-IV-TR includes the diagnosis of Shared Psychotic Disorder, and does not specifically indicate that individuals with this condition have an internal syndrome. It might therefore be relevant to clarify this point in DSM-V.)

\section{Criterion $B$}

DSM-IV notes that mental disorders are associated with distress, disability, or a significantly increased risk of suffering death, pain, disability, or an important loss of freedom. It also gives an example of distress and defines disability as impairment in one or more important areas of functioning.

We recommend that distress and impairment in functioning be retained in criterion B. Psychological distress is central to many mental disorders, especially those considered 'internalizing disorders' (such as depression and anxiety disorders). Including disability in this criterion is needed to identify individuals who need treatment but whose symptoms may not cause them emotional distress. Indeed, it may be argued that distress and disability are not merely associated with the symptoms of a disorder, but they are a result of the disorder, and we suggest emphasizing this causal relationship (Spitzer \& Williams, 1982; Wakefield, 1992).

The definition notes that disability consists of impairment in one or more important areas of functioning; these areas include domains such as occupational, academic, social (including interpersonal), and role functioning. (One set of disorders in DSM-IV-TR that might not, at first glance, be considered to be characterized by distress or impairment are the paraphilias. However, it could be said that symptoms of paraphilias reflect a disturbance in interpersonal functioning.) Because distress and impairment in functioning can vary in terms of degree and severity (i.e. they are dimensional constructs), we suggest 
modifying these terms with the phrase 'clinically significant' to help differentiate impairment indicative of a disorder from milder distress or difficulty in functioning that may not warrant clinical attention or treatment.

This 'clinical significance criterion' (Spitzer \& Wakefield, 1999) has been subjected to criticism when used as one of the operational criteria for individual disorders. One criticism is that this criterion is not widely used in other areas of medicine and is difficult to operationalize (e.g. distress is a highly subjective construct). Nevertheless, we would argue that medical disorders typically implicitly require a judgment that the condition is distressing (e.g. painful) or impairing in some way. Given that we do not have objective biomarkers that adequately define most mental/ psychiatric disorders, the clinical significance criterion remains useful in differentiating disorder from normality.

Regarding the phrase pertaining to 'increased risk', risk factors are important to bear in mind and perhaps even to treat (indeed, the full title of the ICD-10 is International Statistical Classification of Disease and Related Health Problems, with the latter phrase including risk factors for disease such as hypertension); perhaps DSM-V should consider an analogous extension to its title. A full consideration of this issue is beyond the scope of this editorial; diagnosis and treatment of risk factors for psychiatric disorders is appropriately a contentious area, where advantages and disadvantages must be weighed carefully. At the same time, we would note that disorder and risk factors should not be conflated. The phrase 'loss of freedom' can be derived from the concept of disability; that is disability involves one or more losses of freedom (Wakefield, 1992). We therefore tentatively suggest simplifying this criterion by omitting the phrase on risk and on loss of freedom for the sake of clarity. We recognize, however, that limiting the classification to deal with disorders only may be unduly restrictive.

\section{Criterion $C$}

DSM-IV notes that disorders must not be an expectable and culturally sanctioned response to a particular event, for example the death of a loved one. Although it may be difficult to define the term 'expectable', it is important to retain an emphasis on exploring the context of symptoms, and so we suggest retaining this term. Certainly, not all responses to common stressors and losses should be conceptualized as disorders (even if clinical intervention is useful for some of these responses), and we suggest clarifying the criterion to emphasize this point.
The example in DSM-IV of death of a loved one exemplifies the difficulty in reaching a judgment about what is expectable. The boundaries between normal and pathological bereavement are complex and contentious (Kendler et al. 2008). Although bereavement symptoms may be expectable (and culturally sanctioned), studies indicate an association between such symptoms and distress/impairment, and that bereavement symptoms can be modified by clinical intervention. Kendler et al. (2008) noted that the similarities between bereavement-related depression and depression related to other stressful life events substantially outweigh their differences, results that are consistent with a detailed review of the prior literature on this subject (Zisook \& Kendler, 2007).

Part of the context of symptoms is their cultural context. We therefore agree that it is important to retain the idea expressed in the DSM-IV definition that culturally sanctioned responses to events are not considered a mental disorder. An example of this is expectable and culturally sanctioned trance states in religious rituals, and we suggest adding this example in parentheses in criterion $C$.

Although it may be useful for clinicians to distinguish between common responses to stressors and losses (that are distressing but likely to be self-limiting, without high risk of persistent clinically significant distress or impairment) and mental/psychiatric disorders (as defined here), common distressing reactions to common stressors and losses do carry an incremental risk of complications, including the development of mental/psychiatric disorders. Furthermore, people experiencing such normal responses may well present for evaluation and treatment, and they may be helped by a brief intervention such as psychotherapy and monitoring (so that once again, the ICD-10 title of Disease and Related Health Problems has advantages).

\section{Criterion $D$}

DSM-IV refers to behavioral, psychological or biological dysfunction. The term dysfunction can be understood in a statistical way, meaning deviance from a statistical norm (Boorse, 1976), or in an evolutionary framework, meaning deviance from functioning as selected for (Wakefield, 1992). Both of these so-called naturalist approaches are controversial in various ways (Bolton, 2008). One problem with the evolutionary theoretic approach to defining disorder, for example, is that it would involve speculative theoretical assumptions about which syndromes do or do not represent a failure of evolutionary selected psychological or behavioral mechanisms. 
An alternative way of understanding 'dysfunction' is in terms of the consequences of the syndrome, specifically that it leads to or is associated with distress and disability. A related possibility is to define 'dysfunction' as a functioning for the worse, a proposal that requires that the context of symptoms be closely examined and appraised against the patient's life values and goals (Fulford, 1999). Also, it has been argued that the notion of 'dysfunction' draws on particular metaphors of disorder; there is no algorithm that specifies fully the use of the term, rather appropriate use requires careful judgment (Stein, 2008). As other authors have also pointed out (Horwitz \& Wakefield, 2007), context is a key issue in determining whether disorder is present (consider, for example, antisocial behavior in the context of adolescent gangs in some urban areas, where it may be adaptive to join a gang, but where this requires participating in behaviors listed in the diagnostic criteria for conduct disorder). A key aspect of context is the developmental stage of the individual; the boundaries between function and dysfunction change over time and might also be viewed differently by different caregivers (e.g. parents versus teachers). Another possibility is to use a different term, such as 'disturbance', rather than 'dysfunction', as it is not associated with particular theories of function, and is used in some diagnostic criteria sets. This would not, however, resolve the difficulties involved in specifying appropriate use of the term.

The concept that a dysfunction is behavioral, psychological or biological may be taken to imply that there are different levels or types of dysfunction. There is a growing awareness of the extent to which all behavior and psychology are dependent upon brain processes, and the extent to which brain changes have complex behavioral and psychological effects. The term 'psychobiological' emphasizes the extent to which these different types and levels of dysfunction are intertwined in reality, and we therefore recommend incorporating it into the criterion.

\section{Criterion $E$}

DSM-IV requires that deviant behavior and conflicts between the individual and society should not be regarded as disorder, unless they can be shown to be a symptom of dysfunction in the individual. This criterion is arguably not strictly necessary, in that criterion D already indicates that there is dysfunction. Nevertheless, because of the difficulties in specifying fully appropriate use of this term, and because psychiatric diagnoses have been used for political purposes in the past and potential future misuse cannot be ruled out, we suggest, as a precaution, retaining the first part of this criterion. To simplify this criterion, we suggest deleting the second part of the DSM-IV definition because the concept of dysfunction in the individual is already covered by prior criteria, and addition of the word 'primarily' more succinctly conveys the intended point.

\section{Criteria $F$ and $G$}

We suggest adding two more criteria to characterize an individual mental/psychiatric disorder. First, any disorder in DSM should have diagnostic validity (criterion F), on the basis of a number of key validators (e.g. prognostic significance, evidence of psychobiological disruption, or prediction of response to treatment). Although we conceptually require psychobiological dysfunction (criterion D), in the absence of strong empirical evidence for this, other evidence of diagnostic validity is helpful. Evidence for diagnostic validity of different conditions is variable, reflecting in part the amount of research that has been carried out on each condition. DSM-IV had an Appendix for disorders requiring further research, and this provides a place for disorders with weaker validating evidence and may encourage such validation; we would therefore argue for retaining such an appendix in DSM-V, and possibly expanding it with poorly validated DSMIV categories.

Second, any disorder in DSM should have clinical utility (criterion G) (First et al. 2004). That is, we suggest that receipt of a DSM-V diagnosis needs to convey something important about that individual that is relevant in a treatment setting. Our diagnoses should 'do work in the world' and provide useful information about individuals so diagnosed (Kendler, 1990). Diagnosis should facilitate the process of patient evaluation and treatment rather than hinder it. In this regard, considerations of clinical utility may vary from setting to setting; DSM-V requires a balancing of such considerations, so that optimal utility is achieved across more specialized settings and primary care settings.

\section{Criteria $H, I$ and J}

DSM-IV usefully notes that no definition perfectly specifies precise boundaries for the concept of mental/ psychiatric 1 disorder. A large philosophical literature supports this point (Fulford et al. 2006; Stein, 2008) and we agree with retaining this part of criterion $\mathrm{H}$. However, we would add that no definition of which we are aware perfectly specifies precise boundaries for the concept of non-psychiatric medical disorder either.

Ongoing discussions address how best to organize the DSM classification. In criterion I, we have noted 
that considerations about diagnostic validation and clinical utility should help to differentiate disorders from diagnostic 'nearest neighbors'.

The issue of the value-laden nature of defining disorders has received a good deal of attention in the philosophical literature (Fulford, 1989; Sadler, 2005; Bolton, 2008). We suggest acknowledging in criterion J that values inform nosological decisions and specifying that potential benefits should outweigh potential harms when considering whether to add a mental/ psychiatric disorder to, or delete a mental/psychiatric disorder from, the nomenclature.

\section{Conclusion}

The explicit DSM-IV position that mental/psychiatric disorders cannot easily be precisely operationally defined seems, on the one hand, to be basically correct. On the other hand, the position of the DSM process, that our classification system can improve over time as the scientific knowledge base progresses, also seems correct. The situation in psychiatry is reminiscent of some other areas of medicine, where there are also shifting boundaries between normality and abnormality, with evidence-based changes made over time. It is also redolent of many areas of biology, where there may be fuzzy boundaries between constructs (e.g. species), again with evidence-based advances in classification made over time (Stein, 2008; Kendler, 2009).

Contrasting philosophical stances regarding nosological issues have been identified previously, for example contrasting objectivist and evaluativist, internalist and externalist, entity and agent, and categorical and dimensional perspectives (Zachar \& Kendler, 2007). The approach taken here perhaps takes a middle course through some of these debates. For example, we would argue that although gaps in current science mean that a descriptivist position is important (focusing on the symptoms and course of a disorder, rather than merely on its underlying mechanisms), current understandings of psychobiology may usefully inform certain nosological decisions. Disorders cannot be perfectly defined in necessary and sufficient terms, and there are likely to be particularly robust disagreements about more atypical categories. At the same time, disorders are more than mere 'labels', and progress towards a more scientifically valid and more clinically useful nomenclature is possible. Similarly, we hope that our proposals here, although not providing an absolute definition of mental/psychiatric disorder, do help to progress the debate towards a more scientifically valid and more clinically useful definition.

\section{Acknowledgments}

We thank Dr R. Lewis-Fernandez, Dr D. Pine and other members of the DSM-V workgroups who commented on an earlier version of this editorial. Derek Bolton is supported by the NIHR Biomedical Research Centre for Mental Health at the South London and Maudsley NHS Foundation Trust and Institute of Psychiatry, King's College London; and the Centre for Humanities and Health, King's College London.

\section{Declaration of Interest}

Professor Stein has received research grants and/or consultancy honoraria from AstraZeneca, Eli-Lilly, GlaxoSmithKline, Jazz Pharmaceuticals, Johnson \& Johnson, Lundbeck, Orion, Pfizer, Pharmacia, Roche, Servier, Solvay, Sumitomo, Takeda, Tikvah, and Wyeth, and is supported by the Medical Research Council of South Africa. Dr Sadler is supported by National Institutes of Health (NIH) ClinicalTranslational Science Initiative Grant No. KL2 RR024983-01.

\section{References}

Bolton D (2008). What is Mental Disorder? An Essay in Philosophy, Science, and Values. Oxford University Press: Oxford.

Boorse C (1976). What a theory of mental health should be. Journal of the Theory of Social Behaviour 6, 61-84.

First MB, Pincus HA, Levine JB, Williams JBW, Ustun B, Peele R (2004). Clinical utility as a criterion for revising psychiatric diagnoses. American Journal of Psychiatry 161, 946-954.

Fulford KWM (1989). Moral Theory and Medical Practice. Cambridge University Press: Cambridge.

Fulford KWM (1999). Nine variations and a coda on the theme of an evolutionary definition of dysfunction. Journal of Abnormal Psychology 108, 412-420.

Fulford KWM, Thornton T, Graham G (2006). Oxford Textbook of Philosophy and Psychiatry. Oxford University Press: Oxford.

Heyman RE, Smith Slep AM, Beach SR, Wamboldt MZ, Kaslow NJ, Reiss D (2009). Relationship problems and the DSM: needed improvements and suggested solutions. World Psychiatry 8, 7-14.

Horwitz AV, Wakefield JC (2007). The Loss of Sadness: How Psychiatry Transformed Normal Sorrow into Depressive Disorder. Oxford University Press: New York.

Kendler KS (1990). Towards a scientific nosology: strengths and limitations. Archives of General Psychiatry 47, 969-973.

Kendler KS (2009). An historical framework for psychiatric nosology. Psychological Medicine 39, 1-7.

Kendler KS, Myers J, Zisook S (2008). Does bereavementrelated major depression differ from major depression associated with other stressful life events? American Journal of Psychiatry 165, 1449-1455. 
Rounsaville BJ, Alarcón RD, Andrews G, Jackson JS, Kendell RE, Kendler K (2002). Basic nomenclature issues for DSM-V. In A Research Agenda for DSM-V (ed. D. Kupfer, M. B. First and D. E. Regier), pp. 1-29. American Psychiatric Association: Washington, DC.

Sadler J (2005). Values and Psychiatric Diagnosis. Oxford University Press: Oxford.

Spitzer RL, Endicott J (1978). Medical and mental disorder: proposed definition and criteria. In Critical Issues in Psychiatric Diagnosis (ed. R. L. Spitzer and D. F. Klein), pp. 15-39. Raven Press: New York.

Spitzer RL, Wakefield JC (1999). DSM-IV diagnostic criterion for clinical significance: does it help solve the false positive problem? American Journal of Psychiatry 156, 1856-1864.

Spitzer RL, Williams JB (1982). The definition and diagnosis of mental disorder. In Deviance and Mental Illness (ed. W. Grove), pp. 15-31. Sage: Beverly Hills.
Stein DJ (2008). The Philosophy of Psychopharmacology: Smart Pills, Happy Pills, Pep Pills. Cambridge University Press: Cambridge.

van Praag HM (2000). Nosologomania: a disorder of psychiatry. World Journal of Biological Psychiatry 1, 151-158.

Wakefield JC (1992). Disorder as harmful dysfunction: a conceptual critique of DSM-III-R's definition of mental disorder. Psychological Review 99, 232-247.

Wakefield JC, Horwitz AV, Schmitz MF (2005). Are we overpathologizing the socially anxious? Social phobia from a harmful dysfunction perspective. Canadian Journal of Psychiatry 50, 317-319.

Zachar P, Kendler KS (2007). Psychiatric disorders: a conceptual taxonomy. American Journal of Psychiatry 164, 557-565.

Zisook S, Kendler KS (2007). Is bereavement-related depression different than non-bereavement-related depression? Psychological Medicine 37, 779-794. 